\title{
From Quantum to Classical: the Quantum State Diffusion model
}

\author{
N. Gisin, \\ Group of Applied Physics, University of Geneva, 1211 Geneva 4, Switzerland \\ T.A. Brun, \\ Physics Department, Queen Mary and Westfield College, University of London, \\ London E1 4NS, England \\ M. Rigo \\ Mathematics Department, Royal Holloway College, University of London, \\ Egham, Surrey TW20 OEX, England
}

July 31, 2018

\section{Introduction}

The vagueness of the quantum-classical border in physics makes some physicists uncertain about the foundation of their science. Others, by contrast, feel that the accuracy of the verified predictions makes these foundations especially solid. This longtime state of affairs is changing in an interesting way. Recent progress in mesoscopic physics, and the so-called "new science of nanotechnology," open new domains to be explored by theoreticians and experimentalists alike. In this contribution we will exploit numerical and conceptual tools that were developed to explore this new domain of mesoscopic physics, and apply them to systems that are close to the quantum-classical border.

Central to classical mechanics are the concepts of trajectories and the phase space structures associated with them. Hence, we would like to illustrate our results with figures showing these trajectories and structures as they appear in our models. These figures, and the fact that they can be computed without any supplementary parameters, constitute the main result of this contribution.

As examples we could use several kinds of oscillators. The first, and the simplest, is the harmonic oscillator with its regular orbits; but this is boring! Next, systems that exhibit classical chaos, such as the Kicked Anharmonic OScillator (KAOS) [1, 2, 3, , [1] and the double-well Duffing oscillator [5, 6]. In both cases the structure of the strange attractors emerges out of quantum cloudiness when the quantum-classical border is approached. Another example worth mentioning is a nonlinear oscillator exhibiting hysteresis [7], the hysteresis curve appearing when the system becomes more classical.

The feature which characterizes all these systems is that they are open; they interact significantly with some kind of external environment. One cannot in general describe an open system by a single state vector; one requires a density matrix, essentially giving classical probabilities for the system to be in given quantum states. In the limit of Markovian systems, this density matrix evolves according to a linear master equation, as we shall see below.

${ }^{*}$ New address: Institute for Theoretical Physics, University of California, Santa Barbara, CA 93106 U.S.A. 
For many reasons, we would like to get away from the density operator description, and these new tools allow us to do so. These tools require no additional parameters, only a simple idea: describe open quantum systems by state vectors, as for closed systems. Unlike closed systems, their evolution is not unitary - in fact, it is not linear and not deterministic (two general characteristics of open systems). The evolution is chosen such that when the pure state defined by the (normalized) state vector is averaged over the randomness, the usual density matrix description is recovered at all times. The numerical advantage of this approach stems from replacing an $n \times n$ matrix by an $n$ component vector. The conceptual advantage lies in the possibility to have individual states in the theory, as in actual experiments, and to compute mean values as averages of individual outcomes, again as in actual experiments.

This approach shares with the decoherence approach the idea that the environment plays a crucial role in breaking the unitary (boring) evolution and reducing the state 8, 97. However, here the evolution provides classical probabilities for quantum amplitudes. Our approach also shares some similarities with the GRW spontaneous localization model [10], in that the evolution equations are stochastic, but preserve pure states. But here there are no ad hoc parameters.

\section{Quantum State Diffusion and Quantum Jumps}

Markovian open quantum systems are usually described by a master equation:

$$
\dot{\rho}=-i[H, \rho]+\sum_{m}\left(L_{m} \rho L_{m}^{\dagger}-\frac{1}{2}\left\{L_{m}^{\dagger} L_{m}, \rho\right\}\right)
$$

where $\rho$ is the density matrix for the system, $H$ its Hamiltonian, and the linear operators $L_{m}$ describe the effects of the environment. In an unraveling of such master equations, one describes the system in terms of a normalized pure state $|\psi(t)\rangle$ which follows a stochastic "trajectory" in Hilbert space. By averaging the pure state projector $|\psi\rangle\langle\psi|$ over all possible trajectories with appropriate weights, one reproduces the density operator $\rho=M(|\psi\rangle\langle\psi|)$. This is analogous classically to replacing the Fokker-Planck equation for probability densities with a stochastic Langevin equation for single trajectories.

Unfortunately, unlike the case of classical Brownian motion, the unraveling of the master equation (1) is not unique. Thus, there is some ambiguity in how one separates classical and quantum uncertainties, related to the ambiguity in identifying density matrices with quantum ensembles. In this section we consider two well known unravelings of (11).

In quantum state diffusion (QSD), the (Itô) stochastic evolution equation for the normalized state vector $|\psi(t)\rangle$ reads:

$$
\begin{aligned}
|d \psi(t)\rangle= & -i H|\psi(t)\rangle d t-\frac{1}{2} \sum_{j}\left(L_{j}^{\dagger} L_{j}-2\left\langle L_{j}^{\dagger}\right\rangle_{\psi} L_{j}+\left|\left\langle L_{j}\right\rangle_{\psi}\right|^{2}\right)|\psi(t)\rangle d t \\
& +\sum_{j}\left(L_{j}-\left\langle L_{j}\right\rangle_{\psi}\right)|\psi(t)\rangle d \xi_{j}
\end{aligned}
$$

where the "noises" $d \xi_{j}$ are complex-valued Wiener processes of zero mean $M\left(d \xi_{j}\right)=0$ and correlations $M\left(d \xi_{j} d \xi_{k}\right)=0, M\left(d \xi_{j}^{*} d \xi_{k}\right)=\delta_{j k} d t$. This equation describes a continuous nondifferentiable evolution similar to the familiar diffusive paths of a classical Brownian particle, but in Hilbert space instead of real space. QSD is the only continuous unraveling which satisfies the same symmetries as the master equation itself [11, 3].

Our second example is the quantum jumps (QJ) unraveling, which is closely related to photon counting [12, 13]. However, it can be defined for any master equation (1). The stochastic 
increment for the wave-function is

$$
\begin{aligned}
|d \psi(t)\rangle= & -i H|\psi(t)\rangle d t-\frac{1}{2} \sum_{j}\left(L_{j}^{\dagger} L_{j}-\left\langle L_{j}^{\dagger} L_{j}\right\rangle_{\psi}\right)|\psi(t)\rangle d t \\
& +\sum_{j}\left(\frac{L_{j}|\psi(t)\rangle}{\sqrt{\left\langle L_{j}^{\dagger} L_{j}\right\rangle_{\psi}}}-|\psi(t)\rangle\right) d N_{j}
\end{aligned}
$$

The discrete Poissonian noises $d N_{j}$ assume the values 0 or 1 . Most of the time $d N_{j}=0$ and the evolution is continuous and differentiable. However, whenever $d N_{j}=1$ there is a "jump" to the state $L_{j}|\psi(t)\rangle / \sqrt{\left\langle L_{j}^{\dagger} L_{j}\right\rangle_{t}}$. The $d N_{j}$ processes have mean values $M_{|\psi\rangle}\left(d N_{j}\right)=\left\langle L_{j}^{\dagger} L_{j}\right\rangle_{\psi} d t$ and correlations $d N_{j} d t=0$ and $d N j d N_{k}=\delta_{j k} d N_{j}$. This means, essentially, that jumps occur randomly with an average rate $\left\langle L_{j}^{\dagger} L_{j}\right\rangle$.

It has been shown that a quantum jump description of balanced heterodyne detection produces an equation identical to the QSD equation (2) in the limit of a strong local oscillator [14, 15], and that a QSD description of photon detection produces jump-like behavior [11]. Also, in the classical limit (i.e., high photon numbers), the quantum jump equation begins to exhibit diffusive behavior as well [16]. Thus, though these unravelings are distinct, they can behave similarly in certain limits.

\section{Harmonic oscillator}

Let us illustrate these two unravelings for a simple example: the damped harmonic oscillator at finite temperature. $H=\omega a^{\dagger} a, L_{1}=\sqrt{\bar{n} \gamma} a^{\dagger}$ and $L_{2}=\sqrt{(\bar{n}+1) \gamma} a$ where $\bar{n}$ is the thermal equilibrium mean photon number, $\bar{n}=\left\langle a^{\dagger} a\right\rangle_{\rho}$, and $\gamma$ is the inverse relaxation time. For QSD one can show that any initial states tends to a coherent state: $|\psi(t)\rangle \rightarrow\left|\alpha_{t}\right\rangle$, where $a\left|\alpha_{t}\right\rangle=\alpha_{t}\left|\alpha_{t}\right\rangle$ and $\alpha_{t}=\left(\langle q\rangle_{\psi}+i\langle p\rangle_{\psi}\right) / \sqrt{2}$. Furthermore, the evolution of $\alpha_{t}$ is governed by a classical equation,

$$
d \alpha_{t}=-i \omega \alpha_{t} d t-\frac{\gamma}{2} \alpha_{t} d t+\sqrt{\bar{n} \gamma} d \xi_{t}
$$

Hence, for this example at least, the QSD equation fully describes how the environment localizes the quantum state down to a minimum Gaussian wavepacket, and how this wavepacket follows a classical trajectory [17, 18, 19, 20, 21, 22].

For quantum jumps a similar analysis can be carried out, and for a system in a coherent state the evolution of $\alpha_{t}$ is governed by the equation

$$
d \alpha_{t}=-i \omega \alpha_{t} d t-\frac{\gamma}{2} \alpha_{t} d t+\sqrt{\bar{n} \gamma} \frac{\alpha}{\sqrt{|\alpha|^{2}+1}} d W_{t},
$$

where $d W_{t}$ is a real, non-Gaussian stochastic differential variable with mean $M\left(d W_{t}\right)=0$ and $M\left(d W_{t} d W_{t}\right)=d t$, representing the difference between the mean of the jump processes and their actual values. In the classical limit of large $\alpha$, this $d W_{t}$ approaches a diffusion process, and equation (5) has behavior essentially identical to (4).

Somewhat surprisingly, states tend towards small wavepackets for quantum jumps as well as QSD, so the same "classical limit" seems to exist for both [16]. 


\section{Chaotic Duffing oscillator}

A good example of a nonlinear system is the forced, damped Duffing oscillator. This has a classical equation of motion

$$
\frac{d^{2} x}{d t^{2}}+2 \Gamma \frac{d x}{d t}+x^{3}-x=g \cos (t)
$$

and for some choices of $\Gamma$ and $g$ is chaotic [23].

Because the equation of motion includes explicit time-dependence, the solutions lie in a three-dimensional phase space $x, p, t$. It is helpful to consider a discrete surface of section of this system. Let $\left(x_{0}, p_{0}\right)$ be the initial point of the forced, damped Duffing oscillator at time $t_{0}=0$. Then we can define a constant phase map in the $x$-p plane by the sequence of points $\left(x_{n}, p_{n}\right)=\left(x\left(t_{n}\right), p\left(t_{n}\right)\right)$ at times $t_{n}=2 \pi n$. Figure 1 illustrates this in the chaotic regime, where we can clearly see from the surface of section the fractal structure of the strange attractor.

Quantizing the Duffing oscillator is straightforward using the QSD formalism. The Hamiltonian operator is

$$
H(Q, P, t)=P^{2} / 2 m+Q^{4} / 4-Q^{2} / 2+g \cos (t) Q+\sqrt{\Gamma}(Q P+P Q),
$$

and the damping is represented by a Lindblad operator

$$
L=2 \sqrt{\Gamma} a=\sqrt{2 \Gamma}(Q+i P),
$$

where we have assumed $\hbar=1$. The last term in the Hamiltonian is an ansatz, added to give the correct equations of motion in the classical limit; it is necessary due to the simplistic model of the dissipative environment.

This system is far from classical. To go to the classical limit, we introduce a scaling factor $\beta$,

$$
H_{\beta}(Q, P, t)=P^{2} / 2 m+Q^{4} / 4 \beta^{2}-Q^{2} / 2+g \beta \cos (t) Q+\sqrt{\Gamma}(Q P+P Q) .
$$

As we increase $\beta$, the scale of the problem (compared to $\hbar$ ) increases by $\beta$ in $x$ and $p$, without altering the dynamics. Thus, $\beta \rightarrow \infty$ is the classical limit of this system. Classical behavior should emerge from the system in this limit. This is supported by the numerical calculations. (See figures 1 and 2.)

The classical unscaled problem is bounded within a small region of phase space. (See figure 1.) Clearly, the quantized version of this problem (with $\hbar=1$ ) should be far from the classical limit. One would expect to observe little trace of the classical fractal structure. The expectation values of $Q$ and $P$ should be dominated by noise. We solved the forced, damped Duffing oscillator numerically by integrating the QSD equation for three different values of the parameter $\beta$.

Examining figure 2a, we see that the results of the numerical calculation match our expectations very well. The expectation values appear randomly distributed; they are dominated almost completely by the stochastic terms of the equation.

Approaching the classical limit of large $\beta$, more and more of the classical structure of the attractor appears. At first the broad outlines of the attractor are formed, then increasing levels of substructure. (Figures 2b-2c.) The full fractal structure of a strange attractor is only attainable in an unattainable purely classical limit. In an actual physical system, the uncertainty principle provides a lower cutoff to the scale-invariance of the strange attractor.

It can easily be shown that the quantum jumps formalism has a "diffusive limit" similar to QSD as one approaches the classical limit [16]. Thus, exactly the same qualitative behavior is demonstrated in that case as well. 


\section{Conclusions}

Neither QSD nor QJ can be regarded as a fundamental theory, since both divide the universe arbitrarily into a system and environment; and they represent two different and interesting unravelings of the master equation (though only QSD has the same symmetries as the master equation [11, 3]). This is similar to the ambiguity which arises in consistent histories, in which it is possible to choose different sets of histories corresponding to different coarse-grained descriptions 24]. On the other hand, QSD (or QJ) makes testable predictions, and demonstrates the rise of classical physics in a way which is difficult or impossible to see with other theories.

Without entering into a sterile debate, let us note that there seems to be an interesting link between consistent histories and the stochastic models used in this contribution. It appears that the solutions of the stochastic equations provide consistent sets of histories. Hence, it seems that the two approaches are related in a way similar to the relationship in classical mechanics between the global view given by the Maupertuis principle and the local view given by the Newton equations. The global view may be more elegant, but the local one is more useful to compute actual numbers [25, 26].

Quantum mechanics is nonlocal. Classical mechanics is local. Consequently classical mechanics can not explain all quantum phenomena. Conversely, it is cumbersome to use quantum mechanics to describe classical phenomena. Not only are the computations more complex, butand this is the main point - it is conceptually more difficult: one has to argue that nonlocality, entanglement and the principle of superposition can be set aside when crossing the "quantum $\rightarrow$ classical" border. Clearly, nonlocality, entanglement and the principle of superposition should become irrelevant in the classical limit. But why should one argue? Shouldn't it just come out of the equations? Does it come out of the equations? This contribution is about the last question. And the answer is: "it depends on which equation."

\section{Acknowledgments}

We would like to thank Lajos Diósi, Francesca Mota-Furtado, P.F. O'Mahony, Ian Percival and Rüdiger Schack for useful conversations. This research was funded in part by the UK EPSRC, the EU Human Capital and Mobility Program, and the Swiss National Science Foundation.

\section{References}

[1] T.P. Spiller and J.F. Ralph, Phys. Lett. A, 194, 235, (1994).

[2] T.P. Spiller, J.F. Ralph, T.D. Clark, R.J. Prance and H. Prance, J. Low Temp. Phys., 101, 1037 (1995).

[3] N. Gisin and M. Rigo, J. Phys. A, 28, 7375-7390, 1995.

[4] M. Rigo and N. Gisin, Quantum and Semiclass. Optics 8(1), 255, (1996).

[5] T.A. Brun, Phys. Lett. A, 206, 167 (1995).

[6] T.A. Brun, J. Phys. A, 29, 2077 (1995).

[7] M. Rigo, G. Alber, F. Mota-Furtado and P.F. O'Mahony, submitted to Phys. Rev. A.

[8] E. Joos and H.D. Zeh, Z. Phys. B, 59, 223 (1985). 
[9] W.H. Zurek, Physics Today, 36 (October 1991).

[10] G.C. Ghirardi, A. Rimini and T. Weber, Phys. Rev. D, 34, 470 (1986).

[11] N. Gisin and I.C. Percival, J. Phys. A, 25, 5677 (1992); J. Phys. A, 26, 2233 (1993); J. Phys. A, 26, 2245 (1993).

[12] J. Dalibard, Y. Castin, and K. Mølmer, Phys. Rev. Lett., 68, 580 (1992); see also J. Opt. Soc. Am., 10, 524 (1993).

[13] H.J. Carmichael, An Open Systems Approach to Quantum Optics, Lecture Notes in Physics m18, Springer (Berlin 1993).

[14] H.M. Wiseman and G.J. Milburn, Phys. Rev. A, 47, 642 (1993).

[15] P.L. Knight and B. Garraway, in Quantum Dynamics of Simple Systems, eds G.L. Oppo et al., The 44th Scottish University Summer School in Physics, pp 199-237 (1996).

[16] T.A. Brun, N. Gisin, P.F. O'Mahony and M. Rigo, quant-ph/9608038, submitted to Phys. Rev. Letters.

[17] I.C. Percival, J. Phys. A, 27, 1003 (1994).

[18] J.J. Halliwell and A. Zoupas, Phys. Rev. D, 52, 7294 (1995).

[19] R. Schack, T. Brun and I.C. Percival, J. Phys. A, 28, 5401 (1995).

[20] T. Steimle, G. Alber and I.C. Percival, J. Phys. A, 28, L491 (1995).

[21] M. Holland, S. Marksteiner, P. Marte and P. Zoller, Phys. Rev. Lett., 76, 3683 (1996).

[22] M. Rigo and N. Gisin, Quantum and Semiclassical Optics, 8, 255-268 (1996).

[23] M.C. Gutzwiller, Chaos in classical and quantum mechanics, Springer, (Berlin, 1990); J. Guckenheimer and P. Holmes, Nonlinear oscillations, dynamical systems, and bifurcations of vector fields, Springer, (Berlin, 1983).

[24] R. Griffiths, J. Stat. Phys., 36, 219 (1984); R. Omnès, Rev. Mod. Phys., 64, 339 (1992); H.F. Dowker and J.J. Halliwell, Phys. Rev. D, 46, 1580 (1992); M. Gell-Mann and J.B. Hartle, Phys. Rev. D, 47, 3345 (1993).

[25] L. Diósi, N. Gisin, J.J. Halliwell and I.C. Percival, Phys. Rev. Lett., 21, 203 (1995).

[26] T.A. Brun, quant-ph/9606025, submitted to Phys. Rev. Letters.

Figure 1. The constant phase surface of section for the classical forced, damped Duffing oscillator in the chaotic regime, $\Gamma=0.125, g=0.3$.

Figure 2. The constant phase surface of section for a single QSD trajectory of the quantum forced, damped Duffing oscillator in the chaotic regime, $\Gamma=0.125, g=0.3$, for three scalings: a) $\beta=1$, b) $\beta=4$ and c) $\beta=10$. 
Figure 1. The classical Duffing oscillator surface of section.

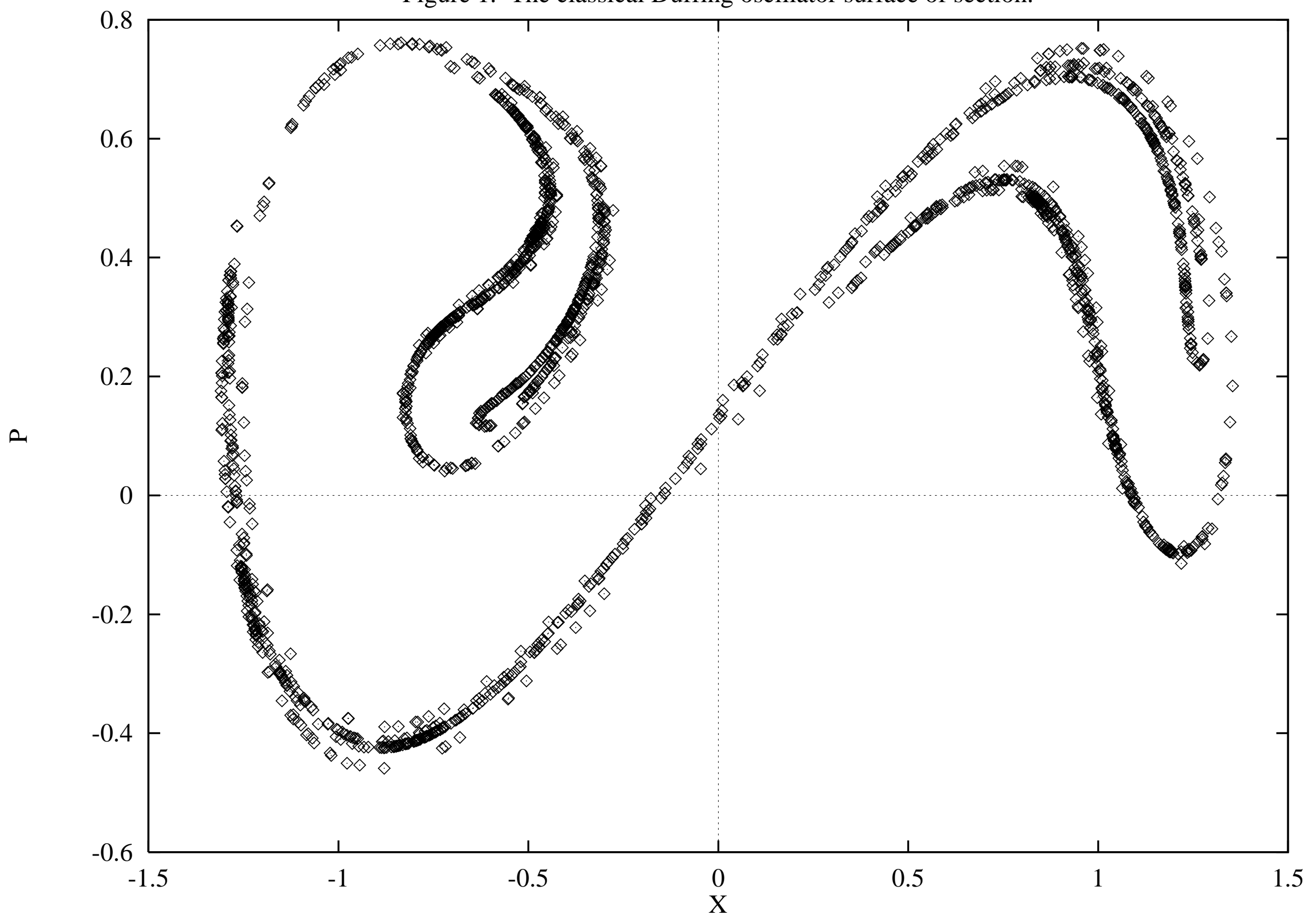




\section{Forced, Damped Duffing Oscillator, Chaotic Regime (QSD) Surface of Section, beta $=1,4,10$}

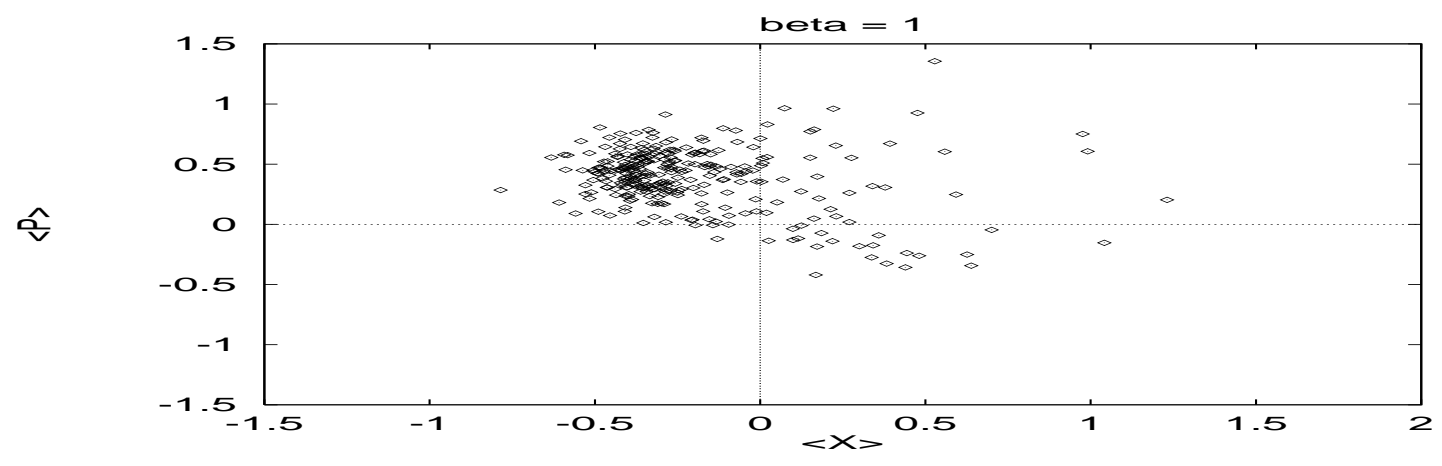

ิิ
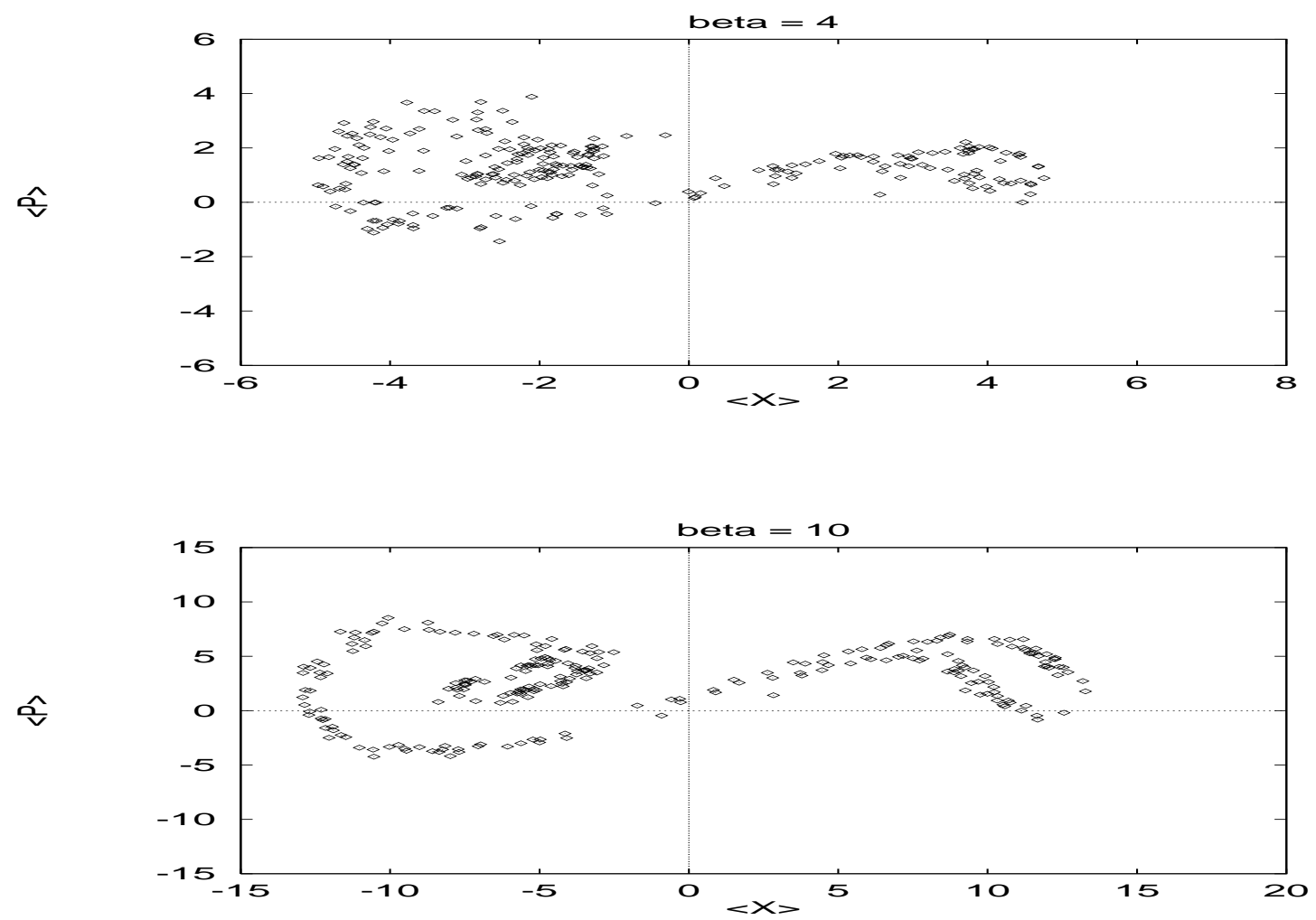